\title{
Liquid Argon Instrumentation and Monitoring in LEGEND-200
}

\author{
Mario Schwarz ${ }^{1(*)}$, Patrick Krause ${ }^{1}$, Andreas Leonhardt ${ }^{1}$, Laszlo Papp ${ }^{1}$, Stefan Schönert ${ }^{1}$, Christoph \\ Wiesinger ${ }^{1}$ \\ ${ }^{(1)}$ Physics Department, Technical University of Munich, Garching, Germany \\ Maria Fomina², Konstantin Gusev², Nadezda Rumyantseva², Egor Shevchik², Daniya Zinatulina² \\ ${ }^{(2)}$ Joint Institute for Nuclear Research, Dubna, Russia \\ Gabriela R. Araujo ${ }^{3}$ \\ ${ }^{(3)}$ Physik-Institut, Universität Zürich, Zurich, Switzerland \\ Corresponding author $(*)$ : mario.schwarz@tum.de
}

\begin{abstract}
LEGEND is the next-generation experiment searching for the neutrinoless double beta decay in ${ }^{76} \mathrm{Ge}$. The first stage, LEGEND-200, takes over the cryogenic infrastructure of GERDA at LNGS: an instrumented water tank surrounding a $64 \mathrm{~m}^{3}$ liquid argon cryostat. Around $200 \mathrm{~kg}$ of Ge detectors will be deployed in the cryostat, with the liquid argon acting as cooling medium, high-purity passive shielding and secondary detection medium. For the latter purpose, a liquid argon instrumentation is developed, based on the system used in GERDA Phase II. Wavelength shifting fibers coated with TPB are arranged in two concentric barrels. Both ends are read out by SiPM arrays. A wavelength shifting reflector surrounds the array in order to enhance the light collection far from the array. The LLAMA is installed in the cryostat to permanently monitor the optical parameters and to provide in-situ inputs for modeling purposes.

The design of all parts of the LEGEND-200 LAr instrumentation is presented. An overview of the geometry, operation principle, and off-line data analysis of the LLAMA is shown.
\end{abstract}

Keywords-liquid argon, silicon photomultiplier, optical monitoring

\section{INTRODUCTION}

$\mathrm{O}$ $\mathrm{NE}$ outstanding question bridging the fields of cosmology and particle physics is the matter-antimatter asymmetry of the universe. This question can not be solved by any mechanism provided by the standard model (SM) of particle physics. According to many theories, the violation of the lepton number conservation can yield an explanation for the asymmetry via leptogenesis [1]. Most of such predict the neutrino to have a Majorana mass component and thus be their own antiparticles [2]. By searching for the hypothetical neutrinoless double beta $(0 v \beta \beta)$ decay, the Majorana mass of the neutrino can be probed and the existence of this decay would imply the violation of lepton number conservation [3].

The transition of a nucleus with mass number $A$ and charge $\mathrm{Z}$ to a nucleus with the same $\mathrm{A}$, but $\mathrm{Z} \pm 2$ is referred to as double beta $(\beta \beta)$ decay. It can only be detected in certain isotopes, where the single beta decay is suppressed [4, 5]. The SM allows for the double beta decay with the emission of two neutrinos since the lepton number is conserved:

$$
(A, Z) \rightarrow(A, Z+2)+e^{-}+e^{-}+\overline{v_{e}}+\overline{v_{e}}(1)
$$

This process is rare due to being a second order process, and has been observed in 11 nuclei so far, including ${ }^{76} \mathrm{Ge},{ }^{82} \mathrm{Se}$, ${ }^{100} \mathrm{Mo},{ }^{130} \mathrm{Te}$ and ${ }^{136} \mathrm{Xe}$ [6].

In contrast, the $0 v \beta \beta$ decay is forbidden in the SM as the lepton number changes by two units:

$$
(A, Z) \rightarrow(A, Z+2)+e^{-}+e^{-}
$$

These two processes can be distinguished experimentally by measuring the summed energy of the two electrons. The $2 v \beta \beta$ decay leads to a continuous spectrum, since the neutrinos carry some of the energy, while electrons produced by the $0 v \beta \beta$ decay share the entire decay energy: $Q_{\beta \beta}$.

The usage of high-purity germanium (HPGe) detectors for the detection of the $0 v \beta \beta$ decay takes advantage of the clear signature in the energy domain by utilizing the superior resolution of this detector type. Enriched in ${ }^{76} \mathrm{Ge}$, HPGe detectors have a high detection efficiency, since source and detector coincide. Their intrinsic radio-purity and the possibility for topology discrimination enable a quasibackground-free search [7].

LEGEND (Large Enriched Germanium Experiment for Neutrinoless $\beta \beta$ Decay) is the next-generation experiment using HPGe detectors to search for the $0 v \beta \beta$ decay. The collaboration is formed by members of the GERDA (GERmanium Detector Array) and MAJORANA collaborations as well as other institutions, thus combining the technical expertise of the field.

The LEGEND collaboration pursues a staged approach, with LEGEND-200 operating $200 \mathrm{~kg}$ of Ge detector material and the subsequent LEGEND-1000 stage operating around one ton of enriched HPGe detectors. The latter aims for a discovery potential at a half-life beyond $10^{28} \mathrm{yr}[8,9]$.

This work focuses on LEGEND-200, which is currently being set up at Laboratori Nazionali del Gran Sasso (LNGS) in Italy using the existing GERDA infrastructure. A sensitivity of $10^{27} \mathrm{yr}$ is aimed for, which can only be reached by achieving a quasi-background-free search. The choice of newly developed inverted coaxial point contact (ICPC) detectors as well as 
improved front-end electronics reduces the background with respect to the precursor experiments. Additionally, the use of electroformed copper will mitigate the background due to structural parts close to the array. The rock overburden of $3500 \mathrm{~m}$ water equivalent and the instrumented water tank surrounding the LAr cryostat reduce both the cosmic muon flux and external gamma radiation [10].

LEGEND-200 will deploy a light readout instrumentation into the scintillating liquid argon (LAr) volume surrounding the Ge detectors. It is designed as an improved version of the LAr instrumentation of GERDA, which enabled this precursor experiment to operate in the quasi-background-free regime during its complete Phase II data taking campaign [10].

\section{THE LIQUid ARGON INSTRUMENTATION OF GERDA AND LEGEND-200}

\section{A. The performance of the GERDA LAr instrumentation}

GERDA deployed a light readout instrumentation in its $64 \mathrm{~m}^{3}$ LAr volume since the beginning of Phase II in 2015. The LAr readout system was upgraded in 2018.

Two sub-systems constituted the LAr instrumentation of GERDA: 3" photomultiplier tubes (PMTs) (nine above the Ge detector array and seven below) as well as wavelength shifting fibers connected to silicon photomultipliers (SiPMs) surrounding the Ge array [10,11]. Fig. 1 shows the energy spectrum in the full GERDA exposure in the region of the $2 v \beta \beta$ continuum. After applying the LAr veto, the remaining events are in agreement with the expectation of a pure $2 v \beta \beta$ sample, as indicated by the blue curve.

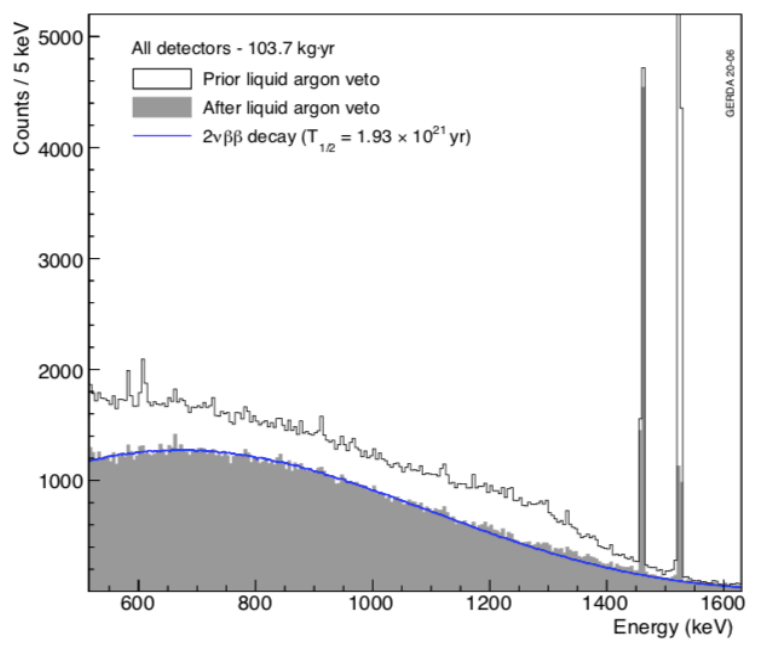

Fig. 1. Energy spectrum measured in GERDA prior (white) and after (grey) LAr veto applied. Both the ${ }^{40} \mathrm{~K}$ and ${ }^{42} \mathrm{~K}$ lines are visible (left/right). The blue line shows the expectation of a pure $2 v \beta \beta$ sample indicating the superior performance of the instrumentation.

Due to its complementarity to pulse shape discrimination (PSD), the LAr instrumentation becomes a powerful tool to reduce the background index in the region of interest (ROI) for the $0 v \beta \beta$ decay search.

For the full Phase II exposure of $103.7 \mathrm{~kg}$ yr the background index (BI) in the ROI in units of cts / ( $\mathrm{keV} \mathrm{kg} \mathrm{yr)} \mathrm{is} \mathrm{shown} \mathrm{in}$ table I after application of different quality cuts. After combining LAr veto and PSD, the background is sufficient for the quasi-background-free search as only 0.3 background events are expected within $\left(Q_{\beta \beta} \pm 2 \sigma\right)$ [10]. The GERDA LAr instrumentation worked stable for more than four years of data taking.

TABLE I

BACKGROUND INDICES IN GERDA

$\begin{array}{ll}\text { prior high-level cuts } & 1.4 \cdot 10^{-2} \\ \text { after PSD } & 3.3 \cdot 10^{-3} \\ \text { after LAr veto } & 5.8 \cdot 10^{-3} \\ \text { after PSD \& LAr veto } & 5.2 \cdot 10^{-4}\end{array}$

Background indices in the ROI in GERDA Phase II. All values are in units of cts / ( $\mathrm{keV} \mathrm{kg} \mathrm{yr)}$

\section{B. Design of the LEGEND-200 LAr instrumentation}

The LEGEND-200 LAr instrumentation builds upon the positive experience of the LAr system in GERDA. It aims for improved efficiency to meet the stringent LEGEND-200 background goals, which are about a factor of 3 lower with respect to GERDA [12].

The detection principle is unchanged: energy depositions in the LAr volume lead to scintillation light emission in the VUV (vacuum ultraviolet) region, centered around $128 \mathrm{~nm}$ [13]. Upon hitting a surface covered with the wavelength shifter TPB (tetraphenyl butadiene), the photons are shifted to the optical blue region. A TPB coating is present on the wavelength shifting fibers. Upon collection, the fibers shift the light further to the green region and trap it with two cladding layers. Captured photons are guided to the fiber ends, coupled to SiPMs and are thus detected.

Ketek PM33100T SiPMs are used for LEGEND-200; the same type was employed in GERDA Phase II. Overall, the array design is the same as in Phase II after the upgrade. Nine SiPMs form one array and are read out in parallel (see fig. 2). The SiPMs are packaged at Technical University of Munich (TUM) in order to meet the radiopurity constraints. The base material of the array consists of suprasil, a high purity synthetic fused silica material. Two sputtered aluminum traces connect all SiPMs. The arrays are read out differentially. In total, 58 modules have been produced.

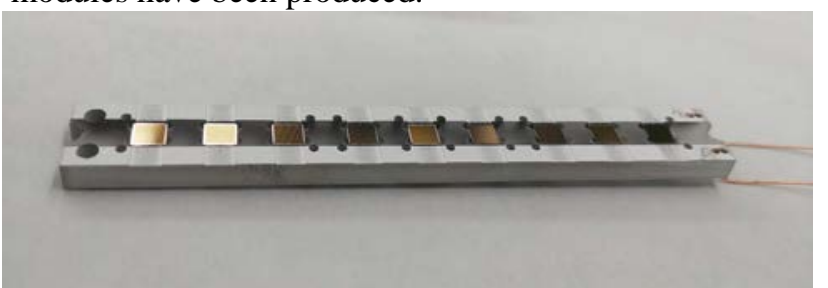

Fig. 2. Array containing 9 SiPMs. Two sputtered aluminum traces connect the SiPMs, which are read out in parallel. The substrate is suprasil, a fused silica material.

The fibers (Saint Gobain BCF 91A) are arranged in two barrels, as shown in fig. 3. All 14 Ge detector strings are located between the concentric barrels.

The outer barrel consists of 20 modules with $1.9 \mathrm{~m}$ long fibers, while the inner one has 9 modules of $1.5 \mathrm{~m}$ long fibers. Each module is made from 81 fibers (nine fibers per each SiPM for nine SiPMs) and is read out at both ends with one SiPM 
array each. This way, the loss of guided light is reduced compared to GERDA, where the fibers were read out at the top only, with an 180 degree bend at the bottom. The fibers at the outer barrel have a 90 degree bend to the inner axis of the array, in order to capture light traveling downwards from the detectors. In contrast to the GERDA instrumentation, no PMTs are present in the argon volume.

The Ge array and the fiber barrels are suspended in the cryostat, filled with $64 \mathrm{~m}^{3}$ of LAr. The rendering in fig. 4 includes also the WaveLength Shifting Reflector (WLSR) surrounding the array, as well as the LEGEND Liquid Argon Monitoring Apparatus (LLAMA) at the bottom of the cryostat (see section III).

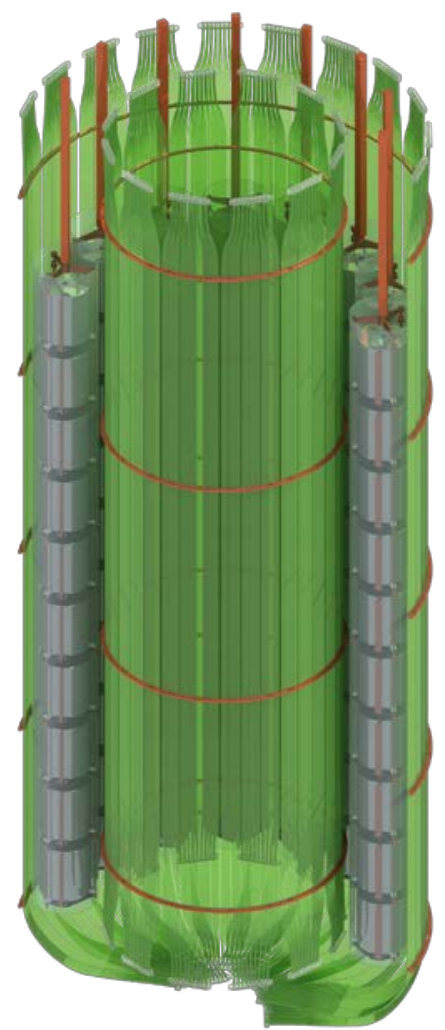

Fig. 3. Rendering of the LEGEND-200 fiber instrumentation, showing the inner fiber barrel, the 14 Ge strings and the outer fiber barrel (the latter two being cut for better visibility).

The WLSR is coated with TPB by evaporation and acts as a reflector for VUV light emissions outside of the fiber barrels. Its radius is $0.7 \mathrm{~m}$. Due to the shifting to the blue region, the photons reflected from the WLSR can reach the fiber assembly without being attenuated in the LAr. Thus, energy depositions outside of the instrumentation, but within $0.7 \mathrm{~m}$ radius can be detected with an increased efficiency. External gamma radiation (e.g. from the cryostat walls), which may Compton scatter in the LAr and deposit energy close to $Q_{\beta \beta}$ in Ge can be mitigated this way.

The WLSR consists of a copper foil with a Tetratex ${ }^{\circledR}$ lining on the inner surface. The evaporation of the TPB was performed in-situ using a dedicated crucible, reaching a TPB thickness of around $600 \mathrm{~nm}$. The performance of a sample of the WLSR was studied at TUM and compared to calibration samples. Fig. 5 shows the resulting photoluminescence spectra under excitation with $128 \mathrm{~nm}$ photons. The performance of the WLSR sample is about twice compared to all reference samples, with small dependence on their thicknesses. This effect is presumably due to the better uniformity achieved in this specific evaporation, with the crucible at a higher temperature and distance with respect to the evaporation of the reference samples.

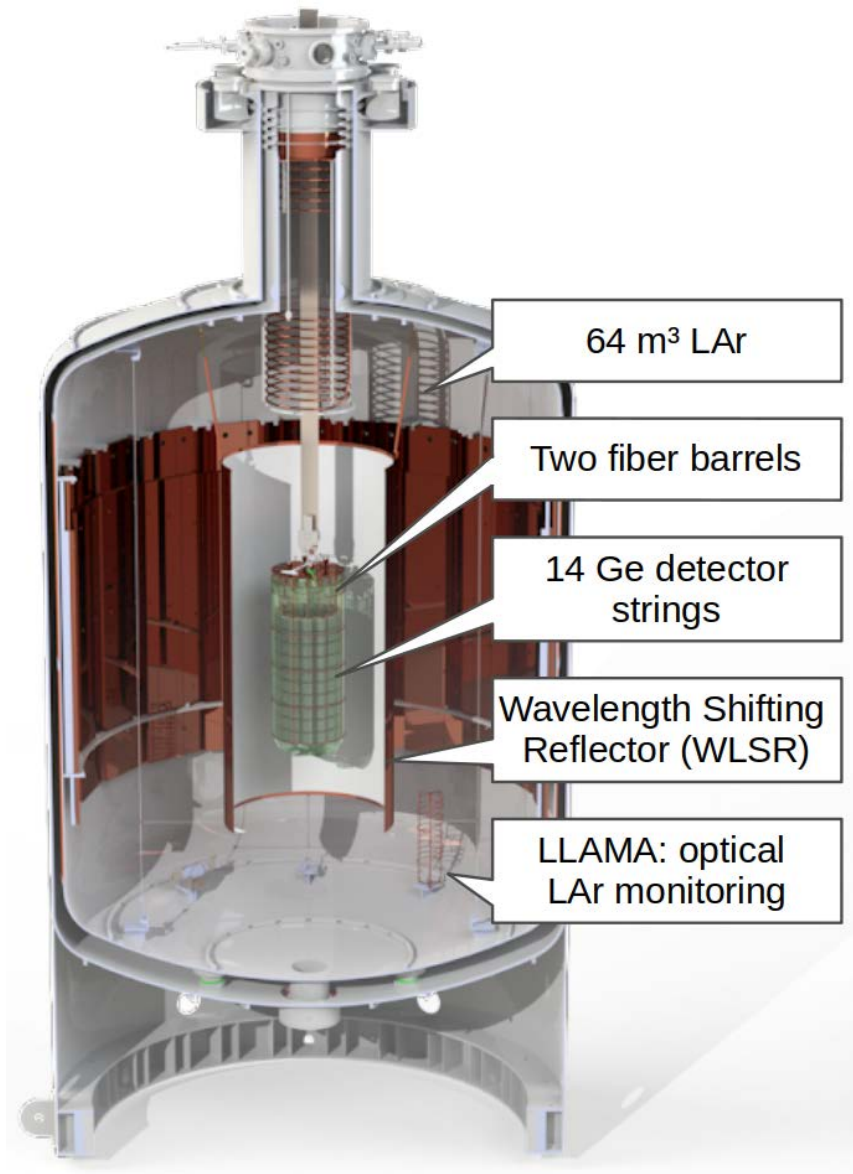

Fig. 4. Rendering of the LEGEND-200 cryostat including the Ge detector array surrounded by the LAr instrumentation (see fig. 3). The WaveLength Shifting Reflector (WLSR) surrounds the array. On the bottom the LEGEND Liquid Argon Monitoring Apparatus (LLAMA) is located (see section III).

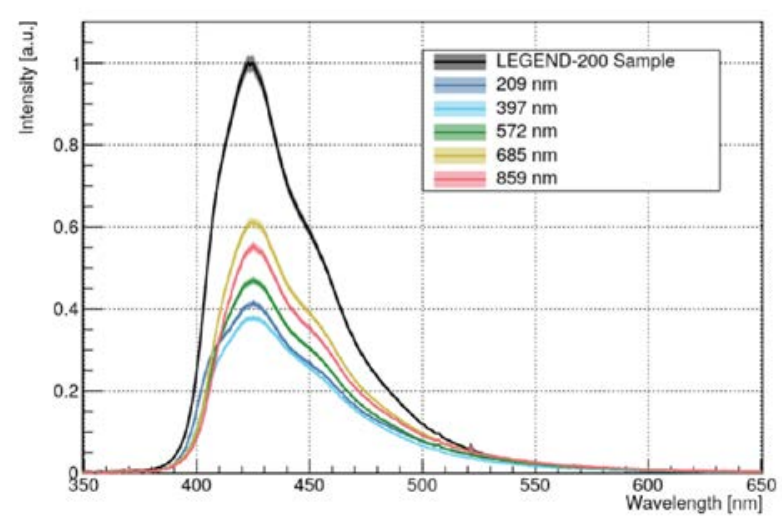

Fig. 5. Photoluminescence spectra under $128 \mathrm{~nm}$ excitation for a WLSR sample as well as Tetratex samples evaporated with TPB at different thicknesses. 


\section{The LEgEND LiQUid ARgON MONITORING ApPARATUS}

\section{A. Motivation}

GERDA and LEGEND-200 - such as any experiment employing a large volume LAr scintillation detector - need a precise understanding of the optical properties of LAr to model the light readout system. Such a model is not ultimately required for the $0 v \beta \beta$ decay search in the mentioned experiments, since no energy is deposited in the LAr for signal events. However, searches for other physics, e.g. $2 v \beta \beta$ shape analyses [14], require a deep understanding of the residual background events and the LAr detector response, especially optical properties of the LAr itself.

One of these parameters is the light yield (LY), i.e. the number of photons emitted per amount of deposited energy. For pure LAr, this value has been measured to be about 41 photons per keV [15].

The time constant of the triplet component of the light emission (triplet lifetime) is another important parameter. Measured values for pure LAr range between $1.3 \mu$ s [13] and $1.6 \mu$ s [16]. Values below $1.3 \mu$ s have been reported, but are nowadays attributed to traces of impurities present in the LAr [17]. Since values at or above $1.5 \mu$ s are measured with the use of TPB, they could be affected by its long emission component [17].

Furthermore, the knowledge of the attenuation length at $128 \mathrm{~nm}$ is required to model the light propagation within the LAr volume. The attenuation length $\lambda_{\text {att }}$ is a combination of the absorption length $\lambda_{a b s}$ and the scattering length $\lambda_{s c a}: 1 / \lambda_{a t t}=$ $1 / \lambda_{\text {abs }}+1 / \lambda_{\text {sca }}$.

Due to the parameters' strong dependence on the actual concentration and composition of impurities in the LAr [18, 19], dedicated measurements within the LAr are required. In order to be able to account for changes of these values over the complete run time of the experiment, a monitor has to be employed, rather than relying on singular measurements. Thus, the LLAMA was constructed and installed in the LEGEND-200 cryostat to measure the three mentioned values continuously and in-situ. The LLAMA was tested and characterized in a $600 \mathrm{l}$ LAr test cryostat located at TUM in Garching. Part of the obtained data is shown in section $\mathrm{D}$.

\section{B. Setup}

A picture of the LLAMA setup is shown in fig. 6. The main structural part of the setup is a $85 \mathrm{~cm}$ high copper frame, holding the light source at the bottom and 13 peripheral SiPMs in the upper part of the setup. The device is operated completely immersed in LAr.

The peripheral SiPMs are located at different distances to the light source, from $15 \mathrm{~cm}$ to $75 \mathrm{~cm}$ with a step size of $5 \mathrm{~cm}$. All of them have individual displacements in the $\mathrm{x} / \mathrm{y}$-plane, to avoid mutual obstructions of the light paths, and are placed in holders with specific inclinations, so the facing directions match their lines-of-sight towards the light source.

The light source (see fig. 7) is a triangular shaped copper vessel containing a triangular shaped cavity. An encapsulated ${ }^{241} \mathrm{Am}$ source is placed within the bottom of the cavity, radiating upwards. The $60 \mathrm{keV}$ gammas create scintillation light events within the cavity.

Three SiPMs are located in the side walls of the cavity and are able to detect the scintillation light. They trigger the data acquisition system and provide primary event information. The open top part of the vessel allows $128 \mathrm{~nm}$ photons to escape, which then travel upwards and might hit a peripheral SiPM. All 16 SiPMs used in the setup are of HAMAMATSU VUV4 type, which are directly sensitive to $128 \mathrm{~nm}$ photons. The lack of wavelength shifters used in the setup reduces systematics as directly the VUV photons are counted.

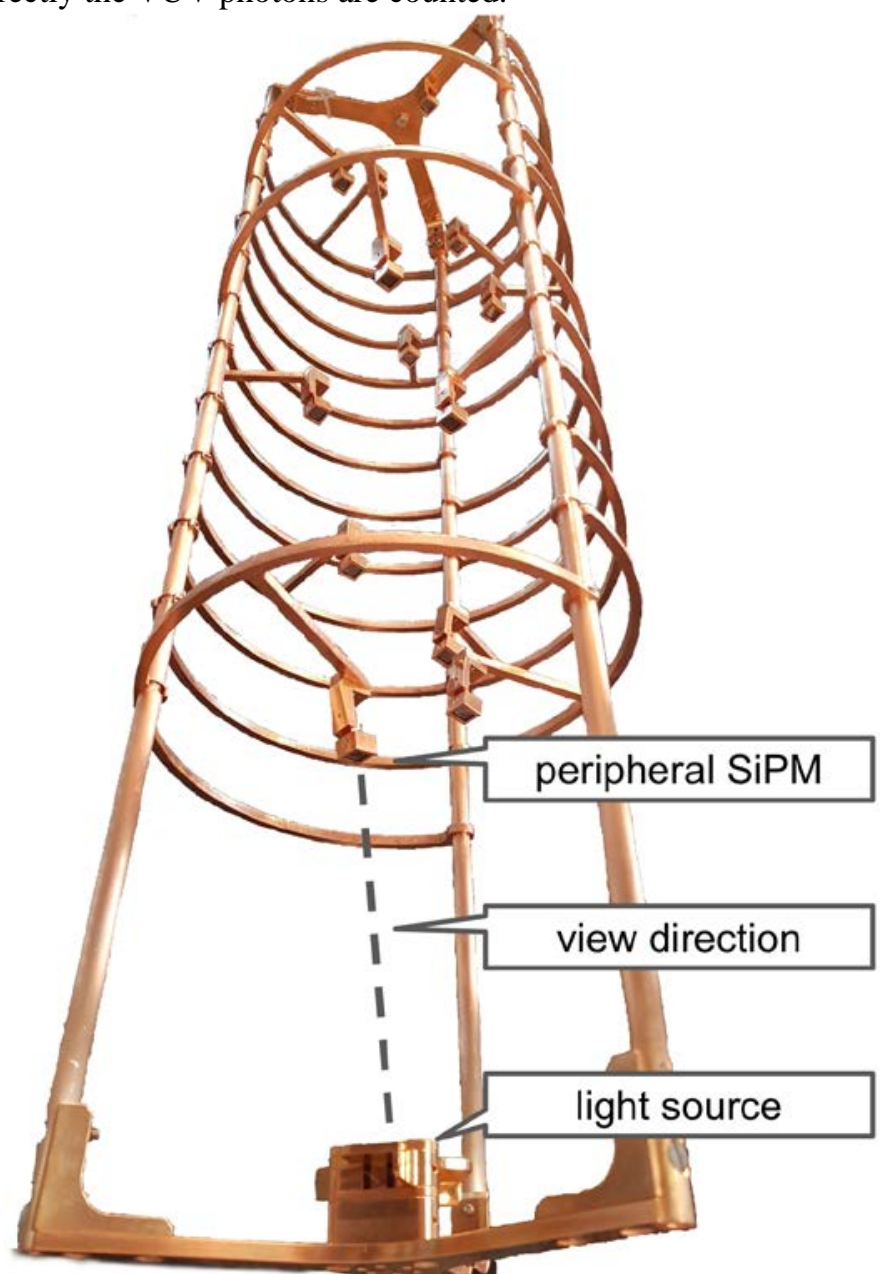

Fig. 6. Picture of the LLAMA setup. The copper structure is about $85 \mathrm{~cm}$ high and holds 13 SiPMs in the top part of the setup as well as the triangular shaped light source, mounted on the bottom plate.

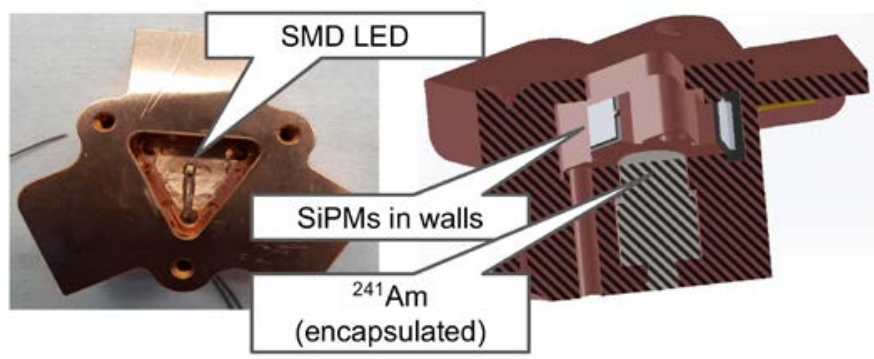

Fig. 7. Photo of the light source (left) and cut view rendering (right). The bottom of the triangular cavity houses both an encapsulated ${ }^{241} \mathrm{Am}$ source and a SMD LED for production of VUV and green photons, respectively. Three SiPMs in the walls yield trigger information. 
A green SMD LED is located at the bottom of the light source. It can be pulsed from outside, in order to calibrate the setup for geometrical as well as photodetection efficiencies of the peripheral SiPMs. This is possible, since visible light is neither affected by absorption, nor scattering effects within LAr.

\section{Readout electronics}

All 16 SiPMs are connected to a power supply and amplification module, providing individual bias voltages as well as amplifying the incoming pulses via Cremat CR-111 charge-sensitive preamplifiers. The amplified pulses are then routed to a Struck SIS3316 FADC (16 input channels, 14 bit resolution) for digitization. A triple coincidence among the three SiPMs located in the source triggers the acquisition of an individual event; all 16 waveforms are read out and stored to a hard drive on this occasion.

\section{Data analysis}

The off-line data analysis of the recorded traces involves the reconstruction of time and charge of incoming pulses via algorithms implemented in the GELATIO framework [20]. Higher-level analysis applied after this step is based on the ROOT framework [21]. SiPM charge spectra are calibrated for photo electron (p.e.) peaks. By summing up the number of p.e. within all three source SiPMs on an event-by-event basis, the scintillation events created by $60 \mathrm{keV}$ gammas become visible as a peak in the spectrum (see fig. 8). The position of this peak is used to reconstruct the relative light yield of the LAr. Quality cuts are applied to obtain mostly events caused by $60 \mathrm{keV}$ gammas for further analysis.

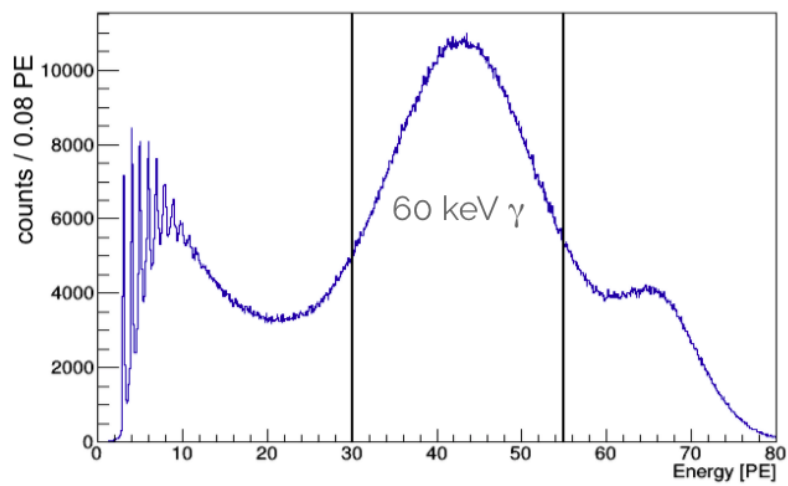

Fig. 8. After summing up the number of photo electrons (p.e.) in the three source SiPMs, the $60 \mathrm{keV}$ peak is obtained. Its position contains information about the light yield in the LAr.

Figure 9 shows the ratio of the amount of light detected by each of the individual source SiPMs, displayed as a ternary plot. Positions of the scintillation events within the source cavity can be reconstructed by this method. This is used to select events happening in the center of the cavity, reducing systematics due to geometrical efficiencies of the peripheral SiPMs.

In contrast to the source SiPMs, detecting multiple photons per event, the occupancy in the peripheral SiPMs is very low, typically equal to or lower than $2 \%$. This allows to reconstruct the light level in these SiPMs via counting analysis. For that, all events are considered, which show 1 p.e. in a given peripheral
SiPM. These events are then counted using two windows in the time domain. Fig. 10 shows an example time spectrum, obtained by calculating the time difference between a pulse within a peripheral SiPM to the time of the scintillation, reconstructed by the source. The two counting windows are indicated by the vertical lines: the blue region counts the valid events (signal), while the red window is used to reconstruct the rate of accidental triggers (background). Furthermore, the triplet lifetime is reconstructed by an exponential fit to the spectrum (red). The result of approximately $1.0 \mu$ s for the LAr in the test cryostat indicates the presence of impurities.

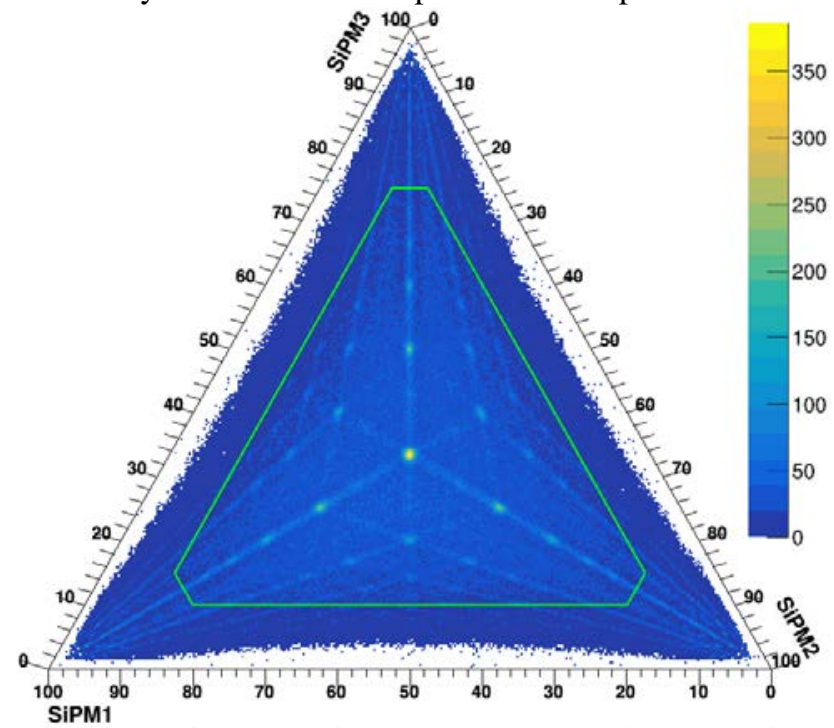

Fig. 9. The relative amount of light detected in each of the three source SiPMs displayed as a ternary plot. This way, it is possible to reconstruct the position of the light emission within the source cavity. The center region (within the lines) is selected by quality cuts.

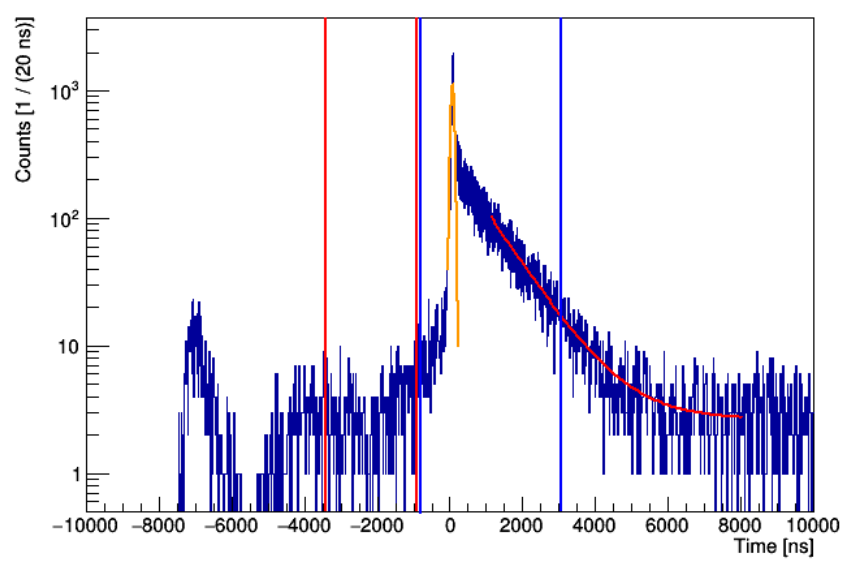

Fig. 10. Time spectrum of the arrival time of pulses in a peripheral SiPM, after subtraction of the time of the beginning of the light emission from the source. The vertical blue and red lines show the signal and background time windows used in counting analysis. The red curve indicates a fit of the triplet component including a flat background contribution.

Using the mentioned counting algorithm, the light level in each of the 13 peripheral SiPMs is reconstructed by dividing the number of 1 p.e. pulses in the signal time window by the number of light emission events in the source, passing all quality cuts. The resulting quantity is referred to as gross PPT (pulse per trigger). After subtraction of the accidental 
background rate, the net PPT is obtained. In fig. 11, a plot of the net PPT values after correction for the individual solid angles is shown. The exponential fit yields an attenuation length of $(50 \pm 4) \mathrm{cm}$ for the LAr in the test cryostat. The quoted uncertainties are statistical only; systematic effects that may cause deviations from the pure exponential behavior are under study.

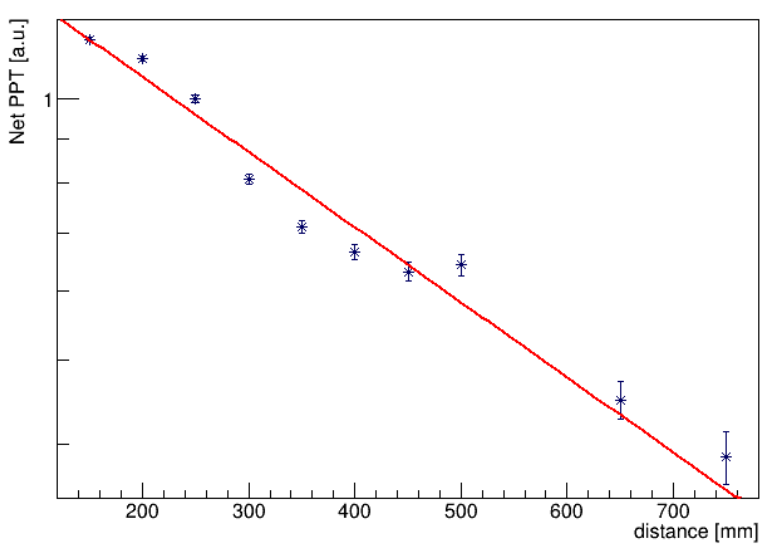

Fig. 11. Attenuation length data obtained from the net PPT values of the individual SiPMs (after correction for the solid angles). The exponential fit yields for this (non-pure) LAr sample an attenuation length of $(50 \pm 4) \mathrm{cm}$.

\section{CONCLUSIONS AND OUTLOOK}

LEGEND-200, the first stage of a next generation experiment searching for the neutrinoless double beta decay, requires more stringent background reduction techniques compared to its predecessor experiments GERDA and MAJORANA Demonstrator, in order to achieve a quasi-background-free operation. Based on the experience in GERDA, an improved light readout instrumentation for detecting scintillation light in the LAr volume surrounding the Ge detectors was designed and constructed. It features an improved optical coverage due to the two-barrel geometry, as well as more efficient light readout due to the placement of the SiPM arrays on both sides of the barrel. The WLSR enhances the light readout up to $70 \mathrm{~cm}$ radial distance. The instrumentation is currently being installed and commissioned at LNGS. Moreover, the usage of new LAr of better quality with respect to GERDA will enhance the efficiency as well.

In order to permanently measure the optical parameters of the LAr of LEGEND-200, the LLAMA was built and characterized at TUM, and finally installed in the LEGEND cryostat at LNGS. During the testing campaign, the ability to monitor the light yield as well as the triplet lifetime of LAr was confirmed, while some systematics in the attenuation length measurements require a more thorough investigation. The LLAMA started data taking during filling of the LEGEND-200 cryostat; the results will be subject of a separate publication.

\section{ACKNOWLEDGMENT}

The work has been supported in part by the German Federal Ministry for Education and Research (BMBF) Verbundforschung.

\section{REFERENCES}

[1] S. Davidson, E. Nardi, and Y. Nir, “Leptogenesis”, Phys. Rep. 466, 105 (2008).

[2] K. Dick, M. Lindner, M. Ratz, D. Wright, "Leptogenesis with Dirac neutrinos”, Phys.Rev.Lett. 84 (2000) 4039-4042

[3] J. Schechter and J. W. F. Valle, "Neutrinoless double beta decay in SU(2) $\times$ U(1) theories”, Phys. Rev. D 25, 2951 (1982)

[4] E.K. Warburton, "Calculation of the Ca-48 (beta-) 48Sc decay rate", Phys.Rev.C 31 (1985) 1896-1898

[5] H. Heiskanen, M.T. Mustonen, J. Suhonen, "Theoretical half-life for beta decay of Zr-96”, J.Phys.G 34 (2007) 837-843

[6] A. Barabash, "Precise Half-Life Values for Two-Neutrino Double- $\beta$ Decay: 2020 Review”, Universe 6 (2020) 10, 159, ArXiv: 2009.14451 [nucl-ex]

[7] GERDA Collaboration, "Background-free search for neutrinoless double- $\beta$ decay of 76Ge with GERDA", Nature 544, 47-52 (2017).

[8] LEGEND Collaboration, "The Large Enriched Germanium Experiment for Neutrinoless Double Beta Decay (LEGEND)”, in AIP Conf.Proc. 1894 (2017) 1, 020027

[9] LEGEND Collaboration, “The Large Enriched Germanium Experiment for Neutrinoless $\beta \beta$ Decay: LEGEND-1000 Preconceptual Design Report”, ArXiv: 2107.11462 [physics.ins-det]

[10] GERDA Collaboration, "Final Results of GERDA on the Search for Neutrinoless Double- $\beta$ Decay”, Phys.Rev.Lett 125, 252502 (2020).

[11] J. Janicskó Csáthy, T.Bode, J. Kratz, S. Schönert, Ch. Wiesinger, "Optical fiber read-out for liquid argon scintillation light”, ArXiv: 1606.04254 [physics.ins-det]

[12] APPEC Committee, “Double Beta Decay APPEC Committee Report”, ArXiv: 1910.04688 [hep-ex]

[13] T. Heindl, T. Dandl, M. Hofmann, R. Krucken, L. Oberauer, W. Potzel, J. Wieser, A. Ulrich, “The scintillation of liquid argon”, EPL 91 (2010) 6, 62002

[14] GERDA Collaboration, "Results on $\beta \beta$ decay with emission of two neutrinos or Majorons in 76Ge from GERDA Phase I”, Eur.Phys.J.C 75 (2015) 9, 416, ArXiv: 1501.02345 [nucl-ex]

[15] T. Doke, et al. "Let Dependence of Scintillation Yields in Liquid Argon”, Nucl.Instrum.Meth.A 269 (1988) 291-296

[16] A. Hitachi, T. Takahashi, N. Funayama, K. Masuda, J. Kikuchi, and T. Doke, "Effect of ionization density on the time dependence of luminescence from liquid argon and xenon”, Phys. Rev. B 27, 5279 (1983)

[17] DEAP Collaboration, "The liquid-argon scintillation pulseshape in DEAP-3600”, Eur.Phys.J.C 80 (2020) 4, 303

[18] A. Neumeier, M. Hofmann, L. Oberauer, W. Potzel, S. Schönert, T. Dandl, T. Heindl, A. Ulrich, and J. Wieser, “Attenuation of vacuum ultraviolet light in liquid argon”, Eur.Phys.J.C 72 (2012) 2190

[19] WArP Collaboration, "Effects of Nitrogen contamination in liquid Argon”, JINST 5 (2010) P06003

[20] M. Agostini, L. Pandola, P. Zavarise, O. Volynets, “GELATIO: A General framework for modular digital analysis of high-purity Ge detector signals”, JINST 6 (2011) P08013

[21] R. Brun and F. Rademakers, "ROOT - An Object Oriented Data Analysis Framework”, Proceedings AIHENP'96 Workshop, Lausanne, Sep. 1996, Nucl. Inst.Meth. in Phys. Res. A 389 (1997) 81-86. 